\title{
Multi-Frequency Pulsar Polarimetry at High Frequencies
}

\author{
A. von Hoensbroech \\ Max-Planck-Institut für Radioastronomies, Bonn, Germany \\ K. M. Xilouris \\ NAIC, Arecibo Observatory, Arecibo, Puerto Rico
}

\begin{abstract}
A sample of 62 pulsar profiles has been studied with full polarization information at high radio frequencies, aiming at studying the dependency of emission altitude at the deepest layers of pulsar magnetospheres. The emission is consistent with heights ranging between $1-2 \%$ of the light-cylinder-radius, while a slight radius-to-frequency mapping is evident.
\end{abstract}

\section{Introduction}

We present polarimetric observations of pulsars made with the $100 \mathrm{~m}$ Effelsberg radiotelescope, using three different receivers centered at $\lambda 18-21 \mathrm{~cm}, 6 \mathrm{~cm}$ and $3 \mathrm{~cm}$. Very high signal-to-noise ratio profiles with milliperiod time resolution were obtained and an elaborate calibration scheme was applied (Hoensbroech $\&$ Xilouris, 1996a) permitting a sensitive analysis. The polarization profiles were used to determine the emission altitude of pulsars at different frequencies using the method developed by Blaskiewicz et al. (1991) which predicts a time lag between the midpoint of the intensity profile and the steepest point of the position angle swing. The advantage of this method is that absolute emission altitudes can be derived without knowledge of the geometry of the pulsar. This method is sensitive to possible differences between core dominated profiles and conals, as the behavior of the position angle in the central part of the profile is crucial for the derived emission altitude.

\section{Data Analysis and Results}

A time lag between the midpoint of the intensity-profile and the steepest point of the position angle swing is predicted by Blaskiewicz et al. (1991), which is proportional to the emission altitude. To derive the midpoint of the profile the $10 \%$ intensity level of the profile outer edges was used (Hoensbroech \& Xilouris, $1996 \mathrm{~b})$. To obtain the steepest point of the position angle, a Rotating-VectorModel was fitted to the data and the steepest point was then derived analytically. A sample of 62 profiles has been processed in this way and in 39 cases, reasonable fits were possible. In 27 cases, the position angle swing lagged the intensityprofile by more than one sigma. There was only one case, where the profile lagged 
the position angle. The emission appears to originate from a region very close to the pulsar surface $(1-2 \%$ of the light-cylinder-radius). The pulsars have been divided into those with core-emission and those with mainly cone-emission using the classification made by Rankin (1993). Evidence is presented that the central emission originates slightly closer to the surface: average values are $r_{\text {core }}=$ $300 \pm 180 \mathrm{~km}$ for core-dominated and $r_{\text {cone }}=490 \pm 240 \mathrm{~km}$ for cone-dominated pulsars. Using a Kolmogorov-Smirnov-test it was shown that the possibility for the two distributions to be drawn from the same parent distribution is less than $10 \%$. The derived emission altitudes have been compared with those calculated with geometrical methods. Most of the altitudes were consistent within the errors. Further investigation of the RFM was applied by assuming a power law dependence, $r \propto \nu^{\alpha}$. In those cases, where a pulsar was observed at more than one frequencies, the individual RFM was calculated by power-law fits (see Table 1). While individual sources behave in a very different way, most of them

$\begin{array}{ll}\text { Table 1. Radius-to-frequency-mapping exponent } \alpha \\ \text { Pulsar } & \alpha \\ \text { PSR 0355+54 } & -0.37 \pm 0.44 \\ \text { PSR 0525+21 } & +0.29 \pm 0.23 \\ \text { PSR 0540+23 } & -0.11 \pm 0.08 \\ \text { PSR 0740-28 } & +0.24 \pm 0.21 \\ \text { PSR 0809+74 } & -0.64 \pm 0.88 \\ \text { PSR 0823+26 } & -0.56 \pm 0.55 \\ \text { PSR 0919+06 } & -0.52 \pm 0.14 \\ \text { PSR 0950+08 } & -0.21 \pm 0.29 \\ \text { PSR 1133+16 } & -0.54 \pm 0.37 \\ \text { PSR 2021+51 } & -0.24 \pm 0.14\end{array}$

show the tendency of decreasing emission altitudes towards higher frequencies. Distinct exception, PSR B0525+21, where the altitude increases with frequency. There is a large scatter among the derived altitudes which could originate due to a saturation towards higher frequencies. However, it is interesting to consider the average RFM. The emission altitudes have been averaged at each frequency and then fitted by a power law which leads to a dependency $\bar{\alpha}=0.20 \pm 0.11$.

Acknowledgments. We thank R. Wielebinski for the support of this work. We also thank M. Kramer and A. Jessner for their help. Arecibo Observatory is operated by Cornell University, under a cooperative agreement with the National Science Foundation.

\section{References}

Blaskiewicz, M., Cordes, J., Wasserman, J. 1991, ApJ, 370, 643

Hoensbroech, A., Xilouris, K.M. 1996a, A\&A, in prep.

Hoensbroech, A., Xilouris, K.M. 1996b, A\&A, in prep.

Rankin, J.M., 1993, ApJS, 85, 145 